\title{
In vitro antioxidant and pancreatic $\alpha$-amylase inhibitory activity of isolated fractions from water extract of Qingzhuan tea
}

\author{
Qian Cheng • Shengbao Cai • Dejiang Ni • Ruojun Wang • \\ Feng Zhou • Baoping Ji • Yuqiong Chen
}

Revised: 10 January 2013 / Accepted: 4 June 2013 / Published online: 18 June 2013

(C) Association of Food Scientists \& Technologists (India) 2013

\begin{abstract}
In the present work, Qingzhuan tea, a unique dark tea produced by post-fermentation technology, was selected to investigate its antioxidant and pancreatic $\alpha$-amylase inhibiting activities. Water extract of Qingzhuan tea was successively isolated by solvent partitioning procedures to obtain chloroform, ethyl acetate, $n$-butanol, sediment and residual aqua fractions. Of different fractions, the ethyl acetate fraction (QEF) had the highest total polyphenols and catechins contents, demonstrated the strongest DPPH radical scavenging activity and exhibited the greatest inhibitory effect on porcine pancreatic $\alpha$-amylase activity in vitro. Further separation of QEF by a Sephadex LH-20 column generated eight subfractions (QEF1QEF8), with QEF8 being the most active subfraction based on the assays above mentioned. The major active components in QEF8 were identified as catechins EGCG and ECG by LC-MS analysis, with contents of $22.29 \%$ and $11.11 \%$ respectively. Inhibitory effects of catechin standards EGCG and ECG on porcine pancreatic $\alpha$-amylase activity were also observed. In conclusion, Qingzhuan tea or its water extract could be potentially used as complementary therapy ingredients for diabetes treatment through lowering postprandial blood glucose, and catechins EGCG and ECG may be the most efficient components in the water extract.
\end{abstract}

\footnotetext{
Q. Cheng $\cdot$ S. Cai $\cdot$ R. Wang $\cdot$ F. Zhou $\cdot$ B. Ji $(\triangle)$

College of Food Science \& Nutritional Engineering, China

Agricultural University, No.17 Tsinghua East Road,

Haidian District, Beijing 100083, China

e-mail: jbp332@gmail.com

D. Ni $\cdot$ Y. Chen $(\bowtie)$

College of Horticulture and Forestry Science,

Huazhong Agricultural University, No.1, Shizishan Street, Hongshan District, Wuhan, Hubei Province 430070, China

e-mail: chenyq@mail.hzau.edu.cn
}

Keywords Qingzhuan tea · Tea extract · Antioxidant . $\alpha$-amylase $\cdot$ Polyphenol $\cdot$ Column chromatography

\section{Introduction}

Global diabetes cases are increasing rapidly and cost vast amounts of resources around the world. Excessive consumption of carbohydrates is revealed to play causative roles in development of various chronic diseases such as obesity, type 2 diabetes and cardiovascular diseases (CVD) (Ludwig 2002). As the major dietary energy source, starch is mainly digested in the gastrointestinal tract by pancreatic $\alpha$-amylase, which is synthesized and secreted by the pancreatic. Slowing the digestion or breakdown of starch may have beneficial effects on insulin resistance and glycemic index control in diabetes patients (Notkins 2002). Introduction of $\alpha$-amylase inhibitor into diets has been demonstrated to be effective in retarding carbohydrate digestion (Golay et al. 1991). Amylase inhibition has gastrointestinal and metabolic effects that may aid in the treatment of diabetes (Layer et al. 1986). Synthetic hypoglycaemic chemicals can produce serious side effects and are not suitable for use during pregnancy (Gilman et al. 1985). Therefore, isolating more effective and safer hypoglycaemic compounds from natural plants has become research trend. Natural $\alpha$-amylase inhibitor has been identified in various plant sources such as cumin seeds, amaranthus caudatus seeds and mangosteen pericarp (Ani and Naidu 2008; Eng Kiat Loo and Huang 2007; Conforti et al. 2005).

Recent evidence suggests that oxidative stress is the underlying mechanism for diabetes development and diabetic complications (Halliwell and Gutteridge 1989). Oxidative stress results from the imbalance between pro-oxidant and 
antioxidant chemicals and leads to cell and tissue damages. Implications of oxidative stress in the pathogenesis of diabetes are comprehensive, involving oxygen free-radical generation, alteration in antioxidant enzymes and lipid peroxides formation etc. (Moussa 2008). Dietary antioxidants have been proposed to slow the progression and ameliorate diabetes. Grapes and tea, which contain many kinds of phenolic compounds, have been verified to induce an anti-hyperglycemic effect in diabetes animal models (Zunino 2009; Hosoda et al. 2003).

Tea is one of the most widely consumed beverage in the world. Recent studies have suggested that it has numerous beneficial health effects in preventing various chronic diseases such as cancer, diabetes, CVD and obesity (Zhu et al. 2006). Furthermore, the radical scavenging and antioxidant properties of tea polyphenols are frequently cited as important contributors to its health improving mechanisms (Higdon and Frei 2003). Aside from their antioxidant bioactivity, tea polyphenols are also shown to be inhibitors of a-amylase (Hara and Honda 1990; He et al. 2007), which provided support to the finding that consumption of tea decreased utilization of dietary carbohydrates (Zhong et al. 2006).

Qingzhuan tea is categorized as a compressed dark tea as $\mathrm{Pu}$-erh tea with over 100 years of production history. It is made with primary tea, which was classified into two kinds of tea, superface tea and inner tea. Superface tea was prepared with fresh leaves with green stem through the processing of green removing, primary rolling, primary sunning, the second parching, the second rolling, pilefermenting and sun drying. Inner tea was processed with more old fresh leaves with red stem by green removing, rolling, pile-fermenting and sun drying. After cutting and sieving, superface tea and inner tea were steamed for $1.5 \mathrm{~min}$ at $120-130{ }^{\circ} \mathrm{C}$, and then poured into a mold in a certain proportion and order to harden into shape. After cooling, the formed tea were removed to the barn to dry for 15 days at $36^{\circ} \mathrm{C}$. When the content of water declined to 8.5-9 \%, Qingzhuan tea product was obtained. As an indispensable beverage for people living in Sinkiang and Tibet areas where vegetables and fruits are often in shortage, Qingzhuan tea's disease prevention and general health care effects were long believed by people living there. Current publications have reported the in vivo pharmacological efficacy of Qingzhuan tea in obesity control (Chen et al. 2008) and lipid clearance (Chen et al. 2010). However, the in vitro antioxidant activity and inhibitory effect on pancreatic $\alpha$-amylase activity of Qingzhuan tea have not been reported yet.

The objectives of this work were to evaluate the in vitro DPPH radical-scavenging and pancreatic $\alpha$-amylase inhibitory activities of different Qingzhuan tea extracts and chromatographical isolations and tentatively identify bioactive components contributing to their bioactivities.

\section{Materials and methods}

Plant materials

Qingzhuan tea (produced in 1997 and stored for 12 years) supplied by Zhaoliqiao Tea Factory, Xianning city, Hubei province, China., and stored at $-20{ }^{\circ} \mathrm{C}$ before extracting.

Chemicals and reagents

$\alpha$-Amylase from porcine pancreatic and 2-Diphenyl-1picryhydrazyl (DPPH) radical were purchased from Sigma Chemical Co. (St. Louis, MO). The standards of (-)epicatechin (EC), (-)-epigallocate-chin (EGC), (+)-catechin (C), (-)-epicatechin gallate (ECG) and (-)-epigallocatechin gallate (EGCG) were purchased from Fisher Chemical Reagent Co. Ltd., USA. Methanol (chromatographic grade) was purchased from Fisher ChemAlert Guide, New Jersey, USA. Sephadex LH-20 was purchased from Pharmacia Biotech, Sweden. All other chemicals used were of analytical grades.

\section{Preparation of extracts from Qingzhuan Tea}

Qingzhuan tea $(50 \mathrm{~g})$ was minced adequately and then extracted with $1,000 \mathrm{~mL}$ of distilled water at $100{ }^{\circ} \mathrm{C}$ for 7 min while stirring continuously. After cooling, the slurries were filtered through medium speed filter papers under vacuum condition and the supernatant was collected. The residue was extracted once more under the same conditions, and the supernatants were combined. The solution was concentrated to $200 \mathrm{~mL}$ by a rotary evaporator at $55{ }^{\circ} \mathrm{C}$ under reduced pressure. The concentrated water extract was then successively extracted with $600 \mathrm{~mL}$ of chloroform, ethyl acetate, and n-butanol, 3 times per organic solvent. After removal of the organic solvents by vacuum concentration, four fractions were obtained: chloroform, ethyl acetate, $n$ butanol and aqua fractions. The aqua fraction was precipitated with 3 times volume of $95 \%$ ethanol (ethanol/water, 95:5 $\mathrm{v} / \mathrm{v}$ ) for $12 \mathrm{~h}$ at room temperature to get the sediment fraction and the residual aqua fraction.

\section{Chromatography on Sephadex LH-20}

The ethyl acetate fraction of Qingzhuan tea water extract (QEF) was selected for further isolating according to the method described by Jie et al. (Jie et al. 2006) with minor modification. In brief, the Sephadex LH-20 column $(50 \mathrm{~cm} \times 1.6 \mathrm{~cm}$ i.d., GE Healthcare Bio-Sciences AB, Sweden) was previously equilibrated with water. Then the ethyl acetate fraction was dissolved in methanol and loaded to the column. The solution was eluted with gradient elution (began with water, followed by an increase of $10 \%$ per degree, $500 \mathrm{~mL}$ per degree, and 
finally with $500 \mathrm{~mL}$ of $50 \%$ acetone at a flow rate of $1.0-$ $\mathrm{mL} / \mathrm{min}$ ). The eluent was sequentially collected, using a fraction collector, and the absorbance was detected at $280 \mathrm{~nm}$, using a UV spectrophotometer. The QEF was fractionated into eight subfractions assigned as QEF1-8, which were evaluated by in vitro DPPH radical-scavenging activity and inhibitory effect on pancreatic $\alpha$-amylase activity.

Determination of total polyphenols and catechins contents

The measurement of total polyphenol was followed by the China National standard method (The first research institute of China Standards Publisher 2003). In brief, $1 \mathrm{~mL}$ of sample solution was added to $4 \mathrm{ml}$ of ferrous tartrate, then phosphate buffer $(\mathrm{pH}=7.5)$ was added to the mixture to make the volumn up to $25 \mathrm{~mL}$. After mixing well, absorbance was measured with $10 \mathrm{~mm}$ cuvette at $540 \mathrm{~nm}$. Distilled water was used instead of the sample solution as the control. Catechins were determined by high performance liquid chromatography (HPLC) method as described by Zhou et al. (2009). An VARIAN HPLC (Model PROSEAR230,USA) equipped with a photo-diode-array detector was employed in the present assay. The analytical column is Agilent TC- $\mathrm{C}_{18}$ column $(150 \times 4.6 \mathrm{~mm}$ inner diameter, with a particle size of $5 \mu \mathrm{m}$, USA). Mobile phases consisted of methanol (with $0.1 \%$ formic acid, mobile phase $\mathrm{A}$ ) and water (with $0.1 \%$ formic acid). A gradient elution was adopted as follows: $0-1 \mathrm{~min}$, $75 \% \mathrm{~A} ; 1-2 \mathrm{~min}, 75-80 \% \mathrm{~A} ; 2-5 \mathrm{~min}, 80 \% \mathrm{~A} ; 5-10 \mathrm{~min}$, $80-75 \%$ A; $10-17 \mathrm{~min}, 75 \% \mathrm{~A}$. The flow rate and column temperature were maintained at $1.0 \mathrm{~mL} / \mathrm{min}$ and $30{ }^{\circ} \mathrm{C}$, respectively. Samples were dissolved, filtered and injected to the column. The injection volume was set at $20 \mu \mathrm{L}$ and detection wavelength was $280 \mathrm{~nm}$.

\section{DPPH radical scavenging assay}

The DPPH radical-scavenging assay was determined by using a previously reported method with a slight modification (Kondo et al. 2002). The reaction mixture contained $1.0 \mathrm{~mL}$ of $0.15 \mathrm{mM}$ DPPH radical solution dissolved in methanol and $1.0 \mathrm{~mL}$ of various concentrations of samples. The absorbance at $516 \mathrm{~nm}$ was measured after the reaction was kept at room temperature in the dark for $30 \mathrm{~min}$. Reagent solution without test samples was used as the control. The scavenging ability was expressed as $\mathrm{EC}_{50}$, represented the effective concentration providing a $50 \%$ of scavenging rate. A lower $\mathrm{EC}_{50}$ value means higher $\mathrm{DPPH}$ radical-scavenging activity.

Pancreatic $\alpha$-amylase inhibition assay

The inhibitory activity of samples against pancreatic $\alpha$-amylase was assayed with an iodine-starch kit following the method of
AI-Dabbas et al. (Al-Dabbas et al. 2006). Briefly, $50 \mu \mathrm{L}$ of aqueous solution of the isolated compounds of different concentrations was mixed with $1.0 \mathrm{~mL}$ of starch substrate $(0.4 \mathrm{~g} / \mathrm{L})$ in phosphate buffer ( $\mathrm{pH} 7.0$ ). After $5 \mathrm{~min}$ of incubation at $37^{\circ} \mathrm{C}, 50 \mu \mathrm{L}$ of $\alpha$-amylase solution $(1 \mathrm{mg} / \mathrm{mL})$ was added to the mixture. After the mixture was further incubated for $7.5 \mathrm{~min}, 1.0 \mathrm{~mL}$ of iodine diluent $(0.01 \mathrm{~mol} / \mathrm{L})$ was added to end the reaction and $3.0 \mathrm{~mL}$ of deionized water was added to dilute the solution to an appropriate concentration for measuring the absorbance at $660 \mathrm{~nm}$. Each sample was analyzed in triplicates. The inhibition of $\alpha$-amylase activity in the presence of samples is calculated by the following equations:

Inhibition $(\%)=[1-($ activity test $/$ activity control $)] \times 100 \%$

Inhibitory abilities of Qingzhuan tea fractions on $\alpha$-amylase activity were expressed as their $50 \%$ inhibition concentration $\left(\mathrm{IC}_{50}\right)$. The lower the $\mathrm{IC}_{50}$ value, the higher the activity for inhibiting effect the Qingzhuan tea fractions possessed on $\alpha$-amylase.

Liquid chromatography-mass spectrometry (LC-MS) analysis

Because of its best activity in pancreatic $\alpha$-amylase inhibition and DPPH radical scavenging, QEF8 was further analyzed by LC-MS. In brief, the sample was applied to a $\mathrm{C} 18$ column $\left(5 \mu \mathrm{m}, 150 \mathrm{~mm} * 4.6 \mathrm{~mm}\right.$ i.d., Agilent TC) maintained at $35^{\circ} \mathrm{C}$, and eluted using a gradient of $3 \%$ acetonitrile $(0.5 \%$ formic acid) to $30 \%$ acetonitrile ( $0.5 \%$ formic acid) over $45 \mathrm{~min}$ at a flow rate of $1.0 \mathrm{~mL} / \mathrm{min}$. The injection volume was $10 \mu \mathrm{L}$. The UV spectra were scanned from 190 to $400 \mathrm{~nm}$. Peaks were determined at $275 \mathrm{~nm}$. The MS parameters were set as follows: negative mode; flow rate of dry gas, $40 \mathrm{~L} / \mathrm{min}$; dry temperature, $250^{\circ} \mathrm{C} ; \mathrm{m} / \mathrm{z}, 100-900$; capillary voltage, $3500 \mathrm{v}$; ESI voltage, $10 \mathrm{kv}$; discharge voltage, $124.8 \mathrm{v}$.

Statistical analysis

All the data were expressed as means \pm standard deviation (SD) of three replicates. Significant differences at $p<0.05$ among means were determined using one-way analysis of variance (one-way ANOVA) in SAS system for Windows V8.

\section{Results and discussion}

Total polyphenols and catechins contents of five fractions from Qingzhuan tea crude water extract

Qingzhuan tea is a speciality tea produced with postfermentation technology and characterized by a period of fungal growth during its manufacturing process. Previous study 
by the present authors indicated that levels of total polyphenols and catechins in Qingzhuan tea after fermentation with microorganisms were decreased due to oxidation and degradation under the catalytic effects of endogenous and exogenous enzymes (Chen et al. 2009). In the present study, crude water extract of Qingzhuan tea was divided into five fractions by polarity and the total polyphenols and catechins contents of these fractions were detected (Table 1). Among the five fractions, the ethyl acetate fraction had the highest total polyphenols content, followed by the $n$-butanol fraction and residual aqua fraction, while the sediment fraction and chloroform fraction had the lowest values $(p<0.05)$. Fu et al. applied the liquid-liquid partition to Fuzhuan tea and obtained the same trend (Fu et al. 2008). HPLC analysis indicated different catechin profiles for various fractions. Total catechins include C, EC, EGC, ECG and EGCG. The total catechins content of the ethyl acetate fraction was significantly greater than that in the $n$-butanol fraction $(p<0.05)$, while there was negligible amount of catechin in the chloroform fraction and residual aqua fraction and no detection in the sediment fraction. In the ethyl acetate fraction, the content of ester catechins was 1.53 -fold higher than the non-ester catechins. These results showed that extracts of different solvents from Qingzhuan tea had substantially different chemical compositions and structures and tea polyphenols and catechins were much more soluble in ethyl acetate than in other organic solvents and water.

Compared with green tea, Qingzhuan tea had lower level of polyphenols but higher levels of polysaccharides and thearubigins (Chen et al. 2009). In the fermentation process, ester catechins are decomposed by hydrolysis with the production of non-ester catechins and gallic acid (Zhang et al. 2011). Therefore, Qingzhuan tea may contain higher amount of gallic acid than green tea. In addition, catechins react with each other to generate theaflavins by polymerization, and then theaflavins continue to converge into thearubigins, finally the small molecular polyphenols polymerize into theabrownins with higher molecular weight. Thus, Qingzhuan tea has a high concentration of theabrownins, the main cause of its color. Due to variations of environmental conditons, composition of fermented tea was significantly changed and some new compounds were identified for the first time in Fuzhuan tea and Puerh tea (Ling et al. 2010; Zhang et al. 2009). Qingzhuan tea may also contain other unknown chemicals.

DPPH radical-scavenging activity of five fractions from Qingzhuan tea water extract

In a previous study of the inhibitory effect of Qingzhuan tea on free radicals, the present authors used the in vitro xanthine oxidase and D-deoxyribose-iron system methods and observed that Qingzhuan tea water extract had strong scavenging effects on superoxide anions and hydroxyl radicals (Chen et al. 2009). In the present study, DPPH radical-scavenging assay, which is widely used in determining the hydrogen donating ability of various natural products (Miliauskas et al. 2004; Chen et al. 1999), was employed to measure the antioxidant capacity of different fractions of Qingzhuan tea water extract. Linear regression analysis revealed that all the fractions displayed a good linear relationship between the scavenging rate and the sample concentration. The regression equations, correlation coefficients and $\mathrm{EC}_{50}$ values were listed in Table 2. Compared with the ethyl acetate fraction, the $\mathrm{EC}_{50}$ value of the $n$-butanol fraction was 2.63 -fold higher while the residual aqua fraction, the sediment fraction and the chloroform fraction were 7.40-fold, 8.25-fold and 37.11-fold higher, respectively. The ethyl acetate fraction had the strongest DPPH radical-scavenging activity, followed by the $n$-butanol fraction, while the scavenging activity of the chloroform fraction was the weakest.

Table 1 The contents of tea polyphenols and catechins of different fractions from Qingzhuan tea water extract (mg/g)

\begin{tabular}{|c|c|c|c|c|c|c|c|}
\hline & \multirow[t]{2}{*}{ Total polyphenols } & \multirow[t]{2}{*}{ Total catechins } & \multicolumn{5}{|c|}{ Individual catechin } \\
\hline & & & $\mathrm{C}$ & $\mathrm{EC}$ & EGC & ECG & EGCG \\
\hline Chloroform fraction & $9.8 \pm 0.57 \mathrm{e}$ & $3.6 \pm 0.59 c$ & $3.6 \pm 0.59$ & ND & ND & ND & ND \\
\hline Ethyl acetate fraction & 787. $2 \pm 26.32 \mathrm{a}$ & $331.4 \pm 9.54 \mathrm{a}$ & $86.5 \pm 0.89$ & $21.2 \pm 0.21$ & $23.1 \pm 0.89$ & $53.7 \pm 0.38$ & $146.9 \pm 8.60$ \\
\hline$n$-Butanol fraction & $310.9 \pm 7.25 b$ & $28.1 \pm 0.67 b$ & $19.8 \pm 1.07$ & ND & $6.6 \pm 0.20$ & $1.7 \pm 0.07$ & ND \\
\hline Sediment fraction & $91.6 \pm 3.54 \mathrm{~d}$ & ND & ND & ND & ND & ND & ND \\
\hline Residial aqua fraction & $111.5 \pm 3.13 c$ & $4.9 \pm 0.29 c$ & $4.9 \pm 0.29$ & ND & ND & ND & ND \\
\hline
\end{tabular}

$n=3$, Mean $\pm \mathrm{SD}$

Values with no letter in common are significantly different $(p<0.05)$

C, (+)-catechin; EC, (-)-epicatechin; EGC, (-)-epigallocate-chin; ECG, (-)-epicatechin gallate; EGCG, (-)-epigallocatechin gallate

$N D$ means not detected 
Table 2 Scavenging effects on DPPH and inhibiting effects on pancreatic $\alpha$-amylase activity of Qingzhuan Tea fractions

\begin{tabular}{|c|c|c|c|c|c|c|}
\hline \multirow[t]{2}{*}{ Fractions } & \multicolumn{3}{|c|}{ Scavenging effect } & \multicolumn{3}{|c|}{ Inhibiting effect } \\
\hline & $\mathrm{EC}_{50}(\mu \mathrm{g} / \mathrm{mL})$ & Regression equation & $\mathrm{R}^{2}$ & $\mathrm{IC}_{50}(\mathrm{mg} / \mathrm{mL})$ & Regression equation & $\mathrm{R}^{2}$ \\
\hline Chloroform fraction & $244.6 \pm 24.62$ & $y=0.1049 x+24.3730$ & 0.9667 & no activity & & \\
\hline Ethyl acetate fraction & $6.6 \pm 0.90$ & $y=6.0419 x+10.2290$ & 0.9668 & $4.6 \pm 0.02$ & $y=9.5624 x+5.6776$ & 0.9832 \\
\hline$n$-Butanol fraction & $17.3 \pm 2.89$ & $y=2.0741 x+14.0570$ & 0.9680 & $11.3 \pm 0.32$ & $y=4.7757 x-3.8321$ & 0.9497 \\
\hline Sediment fraction & $54.5 \pm 8.16$ & $y=0.6736 x+13.4080$ & 0.9879 & no activity & & \\
\hline Residial aqua fraction & $48.8 \pm 6.66$ & $y=0.7486 x+13.4940$ & 0.9835 & no activity & & \\
\hline
\end{tabular}

$n=3$, Mean $\pm \mathrm{SD}$

The above results showed that total polyphenols, total catechins and antioxidant activities were significantly different among different isolations. Tea polyphenols and catechins were verified to have antioxidant activities (Higdon and Frei 2003). Correlation analysis between the levels of total polyphenols and catechins and $\mathrm{EC}_{50}$ values were undertaken and both were greatly correlated with DPPH assays $\left(\mathrm{r}^{2}=0.607\right.$ and $r^{2}=0.654$, respectively). Previous studies reported similar correlations between these parameters for green tea and other types of tea products (Fukushima et al. 2009; Anesini et al. 2008; Karori et al. 2010). It was inferred that DPPH radicalscavenging activity was likely due to polyphenols and catechins existed in the Qingzhuan tea extracts.

Inhibitory effect of five fractions from Qingzhuan tea water extract on in vitro pancreatic $\alpha$-amylase activity

$\alpha$-Amylases catalyze the hydrolysis of $\alpha$-1, 4-glucosidic linkage of starch to initiate starch digestion and promote glucose absorption. $\alpha$ - Amylase inhibitors were considered to be effective in diabetes control (Ponnusamy et al. 2011). In this study, an in vitro $\alpha$-amylase inhibition model was used to screen the extracts of Qingzhuan tea to evaluate their potential hypoglycaemic effects.

The $\alpha$-amylase inhibitory activity of Qingzhuan tea fractions was assayed by the method of iodine-starch reaction. As shown in Table 2, no $\alpha$-amylase inhibition was observed in the chloroform fraction, sediment fraction and residual aqua fraction of Qingzhuan tea. The ethyl acetate and $n$ butanol fraction exhibited a dose-dependent inhibitory effect on $\alpha$-amylase activity. The $\mathrm{IC}_{50}$ value of $n$-butanol fraction $(11.27 \mathrm{mg} / \mathrm{mL})$ was 2.43 -fold higher than the ethyl acetate fraction $(4.64 \mathrm{mg} / \mathrm{mL})$, suggesting that the ethyl acetate fraction exhibited greater inhibitory activity than $n$-butanol fraction. A similar situation was found in a study performed on black tea (Kusano et al. 2008).

Based on the above result, we found that the ethyl acetate fraction contained the highest amount of tea polyphenols and had the greatest $\alpha$-amylase inhibitory activity. It's obvious that increasing polyphenols concentration would increase $\alpha$ amylase inhibition. Thompson and Yoon (1984) reached a similar conclusion in their study on starch digestibility affected

Table 3 Scavenging effects on DPPH and inhibiting effects on pancreatic $\alpha$-amylase activityof purification products from the ethyl acetate fraction

\begin{tabular}{|c|c|c|c|c|c|c|}
\hline \multirow[t]{2}{*}{ Subfractions } & \multicolumn{3}{|c|}{ Scavenging effect } & \multicolumn{3}{|c|}{ Inhibiting effect } \\
\hline & $\mathrm{EC}_{50}(\mu \mathrm{g} / \mathrm{mL})$ & Regression equation & $\mathrm{R}^{2}$ & $\mathrm{IC}_{50}(\mathrm{mg} / \mathrm{mL})$ & Regression equation & $\mathrm{R}^{2}$ \\
\hline QEF1 & $360.3 \pm 0.12$ & $y=1.1760 x+7.3522$ & 0.9956 & $>16$ & & \\
\hline QEF2 & $270.1 \pm 10.53$ & $y=7.0445 x-0.1542$ & 0.9984 & $10.9 \pm 0.40$ & $y=5.1760 x-6.3324$ & 0.9974 \\
\hline QEF3 & $235.2 \pm 9.64$ & $y=0.1610 x+12.1430$ & 0.9587 & $9.4 \pm 0.31$ & $y=6.2048 x-8.0609$ & 0.9594 \\
\hline QEF4 & $109.7 \pm 8.73$ & $y=0.4350 x+6.6394$ & 0.9762 & $8.2 \pm 0.25$ & $y=6.3727 x-2.2339$ & 0.9509 \\
\hline QEF5 & $105.4 \pm 0.97$ & $y=0.4274 x+4.9564$ & 0.9926 & $7.9 \pm 0.23$ & $y=4.9771 x+10.5100$ & 0.9932 \\
\hline QEF6 & $81.9 \pm 1.75$ & $y=0.5252 x+7.7326$ & 0.9820 & $2.4 \pm 0.10$ & $y=14.2170 x+16.3680$ & 0.9144 \\
\hline QEF7 & $77.9 \pm 1.32$ & $y=0.5195 x+9.5402$ & 0.9918 & $1.6 \pm 0.04$ & $y=16.6360 x+24.1290$ & 0.9164 \\
\hline QEF8 & $4.6 \pm 0.43$ & $y=10.1880 x+3.6992$ & 0.9821 & $0.4 \pm 0.01$ & $y=163.6900 x-10.1260$ & 0.9717 \\
\hline
\end{tabular}

$n=3$, Mean \pm SD

QEF1-QEF8, the first to eighth subfraction of the ethyl acetate fraction through Sephadex LH-20 column 
Fig. 1 Chromatogram of QEF8 from the ethyl acetate fraction by HPLC. QEF8, the eighth subfraction of ethyl acetate fraction through Sephadex LH20 column



by polyphenols. Other studies suggested that tea polyphenols had the capacity to inhibit $\alpha$-amylase activity (Hara and Honda 1990; He et al. 2007). It was concluded that tea polyphenols might be an important factor in contributing to the $\alpha$-amylase inhibitory activity of Qingzhuan tea extract.

DPPH radical-scavenging activity of eight subfractions from the ethyl acetate fraction

As the ethyl acetate fraction had the highest tea polyphenols and catechins contents, the strongest DPPH radicalscavenging activity and the greatest inhibitory effect on $\alpha$ amylase activity in vitro, further separation with a Sephadex LH-20 column by different concentrations of methanol as mobile phase was performed and generated eight subfractions. Table 3 showed that their $\mathrm{IC}_{50}$ values ranged from 4.55 to $360.27 \mu \mathrm{g} / \mathrm{mL}$, and were in the following order: QEF1 > QEF2 > QEF3 > QEF4 > QEF5 > QEF6 > QEF7 > QEF8. It was concluded that QEF8 exhibited the strongest scavenging effect on DPPH radical, which was consistent with the report of Jie et al.. Eight fractions was also obtained using liquid-liquid partition and column chromatography in Pu-erh tea, and fraction 8 from the ethyl acetate extract was found to display the greatest hydroxyl radical scavenging activity as well (Jie et al. 2006). According to the normal-phase and sizeexclusion chromatographic separation mechanism, QEF8 may have relatively stronger polarity and higher molecular weight than the other fractions.

Inhibitory effect of eight subfractions from ethyl acetate fraction on in vitro pancreatic $\alpha$-amylase activity

The inhibitory potency of Qingzhuan Tea subfractions against $\alpha$-amylase was determined, and their $\mathrm{IC}_{50}$ values were displayed in Table 3. All subfractions showed $\alpha$-amylase inhibition capabilities. The order of their $\mathrm{IC}_{50}$ values was the same with that of DPPH assays, and QEF8 had the highest inhibitory activity against $\alpha$-amylase and the lowest $\mathrm{IC}_{50}$ value of $0.37 \mathrm{mg} / \mathrm{mL}$.

\section{LC-MS analysis of QEF8}

The compounds responsible for antioxidant activity and $\alpha$ amylase inhibition in QEF8 were further analyzed by HPLC and LC-MS method, as this component showed the in vitro greatest DPPH radical scavenging activity and inhibitory effect against $\alpha$-amylase. Figure 1 revealed that HPLC chromatogram of QEF8 had two major peaks, peak 1 and peak 2

Table 4 LC-MS Data for QEF8

\begin{tabular}{lllllll}
\hline Peak No. & Retention time $(\mathrm{min})$ & PDA & Ratio $(100 \%)$ & M/Z (M-H) & MS $^{2}$ & Tentative identification \\
\hline 1 & 16.815 & 275 & 22.29 & $457,305,169$ & $331,305,169$ & EGCG \\
2 & 23.967 & 275 & 11.11 & 441,289 & 289 & ECG \\
\hline
\end{tabular}

QEF8: the eighth subfraction of ethyl acetate fraction through Sephadex LH-20 column; EGCG, (-)-epigallocatechin gallate; ECG, (-)-epicatechin gallate 
Fig. 2 The basic structures of catechins considered in the present study<smiles>O=C(O[C@H]1Cc2c(O)cc(O)cc2O[C@H]1c1cc(O)c(O)c(O)c1)c1cc(O)c(O)c(O)c1</smiles>

(-)-Epigallocatechin gallate<smiles>O=C(O[C@H]1Cc2c(O)cc(O)cc2O[C@H]1c1ccc(O)c(O)c1)c1cc(O)c(O)c(O)c1</smiles>

(-)-Epicatechin gallate with the retention time of $16.815 \mathrm{~min}$ and $23.967 \mathrm{~min}$ respectively. The tentatively identified compounds were depicted in Table 4. The EI spectrum of Peak 1 contains the molecular $[\mathrm{M}-\mathrm{H}]^{+}$ion at $\mathrm{m} / \mathrm{z}=457 \mathrm{Da}$, with prominent fragments at $m / z=331,305$ and $169 \mathrm{Da}$. The EI spectrum of Peaks 2 contains the molecular $[\mathrm{M}-\mathrm{H}]^{+}$ion at $\mathrm{m} / \mathrm{z}=441 \mathrm{Da}$, with prominent fragments at $\mathrm{m} / \mathrm{z}=289 \mathrm{Da}$. The mass ion and the fragment ions of the two peaks were essentially identical to the pattern obtained by Gondoin et al. (Gondoin et al. 2010). Thereby, it is proposed that they were EGCG and ECG accordingly (Fig. 2). Based on the comparison of peak areas with the authentic samples, EGCG and ECG were quantified to account for $22.29 \%$ and $11.11 \%$ respectively in QEF8.

Inhibitory effect of catechin standards (EGCG, ECG) on in vitro pancreatic $\alpha$-amylase activity

Based on the above result, we found that EGCG and ECG were the major active compounds in QEF8. Apart from their antioxidant bioactivity, catechins were reported to inhibit intestinal $\alpha$-amylase or sucrase, deter the digestion of certain amounts of starch or sucrose and eventually reduce the plasma glucose levels in vivo (Matsumoto et al. 1991). To further confirm components in QEF8 that are the most effective as inhibitors, EGCG and ECG were tested on the inhibition of porcine pancreatic $\alpha$-amylase. As shown in Fig. 3, EGCG showed greater inhibitory effect than ECG, which was inconsistent with the result reported by Hara and Honda (1990). The discrepancy may be due to the difference of the $\alpha$-amylase source or the test conditions. The essence of enzyme inhibition herein was protein precipitation through forming various complexes with polyphenols (Siebert et al. 1996) or calcium (required as a cofactor for amylase enzyme activity) binding (Yoon et al. 1983). EGCG had more galloylated groups and higher molecular weight, which may account for its better inhibiting activity.
Earlier studies showed that there are synergistic effects between different catechins. Chung et al. reported that the effect of green tea extract on inhibition of lung tumor genesis was better than that of EGCG alone (Chung et al. 2003). Shi and Kakuda found that their antioxidant activity was enhanced by the synergistic action between catechins, e.g. EGCG, EGC, ECG, EC, pheophytins a and b, and other components in tea leaves (Shi and Kakuda 2006). Yang also discovered that free radical-scavenging was affected by different proportions of catechin monomers, and the best proportion of EGCG:ECG:EGC:EC would be 5:2:2:1 (Yang 2003). Thereby, the inhibitory effect against $\alpha$-amylase of QEF8 may be due to the synergistic action of catechins and other compounds, which demands further research.

In conclusion, Qingzhuan tea exhibited good antioxidant activity and inhibiting effect against pancreatic $\alpha$-amylase, and may be used as oral antidiabetic diet. EGCG and ECG were responsible for the inhibitory activity in Qingzhuan tea extracts.

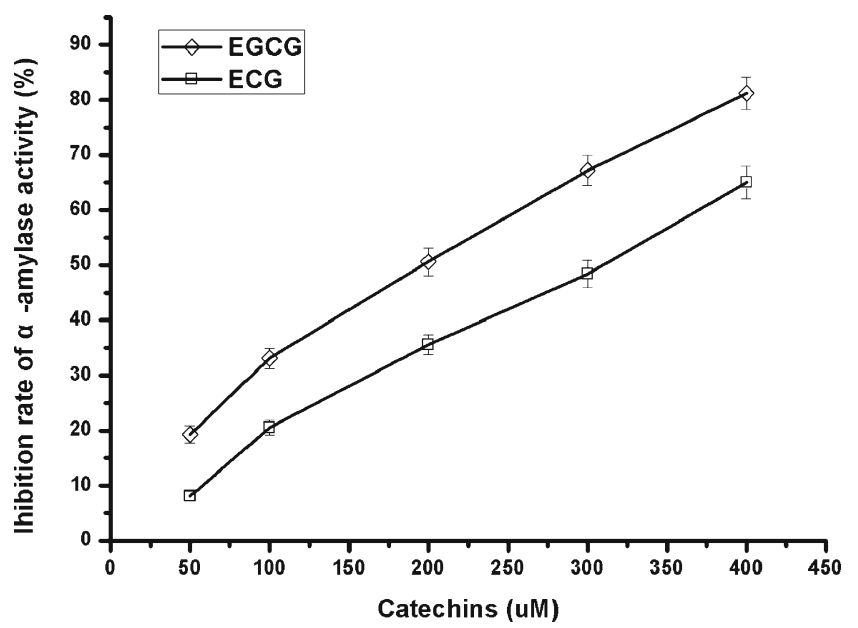

Fig. 3 Inhibitory effects of EGCG and ECG on pancreatic a-amylase activity. EGCG, (-)-epigallocatechin gallate; ECG, (-)-epicatechin gallate. Values are expressed as mean $\pm \operatorname{SD}(n=3)$. 


\section{References}

Al-Dabbas MM, Kitahara K, Suganuma T, Hashimoto F, Tadera K (2006) Antioxidant and alpha-amylase inhibitory compounds from aerial parts of Varthemia iphionoides Boiss. Biosci Biotechnol Biochem 70(9):2178-2184

Anesini C, Ferraro GE, Filip R (2008) Total polyphenol content and antioxidant capacity of commercially available tea (Camellia sinensis) in Argentina. J Agric Food Chem 56(19):9225-9229

Ani V, Naidu KA (2008) Antihyperglycemic activity of polyphenolic components of black/bitter cumin Centratherum anthelminticum (L.) Kuntze seeds. Eur Food Res Technol 226(4):897-903

Chen Y, Wang M, Rosen RT, Ho CT (1999) 2, 2-Diphenyl-1picrylhydrazyl radical-scavenging active components from Polygonum multiflorum Thunb. J Agric Food Chem 47(6):2226-2228

Chen YQ, Zhang W, Cheng Q, Dong JF, Liu XH, Gan DP, Qian YP (2008) The anti-obesity effects of Hubei Qingzhuan tea on rats (Chinese). J Tea Sci 28(5):363-369

Chen YQ, Ni Dj, Cheng Q, Ma R, Kong LQ (2009) Study on the main components and capacities of scavenging free radicals of Qingzhuan tea. Proceedings of the Symposium on technological innovation and industrial development of Tea 427-432

Chen YQ, Zhang W, Ni DJ, Cheng Q, Liu XH, Gan DP (2010) Study on the hypolipidemic effect and antioxidative activity of Hubei Qingzhuan tea (Chinese). J Tea Sci 30(2):124-128

Chung FL, Schwartz J, Herzog CR, Yang YM (2003) Tea and cancer prevention: studies in animals and humans. J Nutr 133(10):3268-3274

Conforti F, Statti G, Loizzo MR, Sacchetti G, Poli F, Menichini F (2005) In vitro antioxidant effect and inhibition of $\alpha$-amylase of two varieties of Amaranthus caudatus seeds. Biol Pharm Bull 28(6):1098-1102

Fu DH, Liu ZH, Huang JA, Chen HH (2008) The effect of different extracts of Fuzhuan tea on the activities of digesting enzyme (Chinese). J Tea Sci 28(1):62-66

Fukushima Y, Ohie T, Yonekawa Y, Yonemoto K, Aizawa H, Mori Y, Watanabe M, Takeuchi M, Hasegawa M, Taguchi C (2009) Coffee and green tea as a large source of antioxidant polyphenols in the Japanese population. J Agric Food Chem 57(4):1253-1259

Gilman AG, Goodman LS, Rail TW, Murad F (1985) The pharmacological basis of therapeutics. Macmillan, New York

Golay A, Schneider H, Temler E, Felber JP (1991) Effect of trestatin, an amylase inhibitor, incorporated into bread, on glycemic responses in normal and diabetic patients. Am J Clin Nutr 53(1):61-65

Gondoin A, Grussu D, Stewart D, McDougall GJ (2010) White and green tea polyphenols inhibit pancreatic lipase in vitro. Food Res Int 43(5):1537-1544

Halliwell B, Gutteridge JMC (1989) Free radicals in biology and medicine. Clarendon, Oxford

Hara Y, Honda M (1990) The inhibition of $\alpha$-Amylase by tea polyphenols. Agric Biol Chem 54(8):1939-1945

He Q, Lv Y, Yao K (2007) Effects of tea polyphenols on the activities of alpha-amylase, pepsin, trypsin and lipase. Food Chem 101(3):11781182

Higdon JV, Frei B (2003) Tea catechins and polyphenols: health effects, metabolism, and antioxidant functions. Crit Rev Food Sci 43(1):89-143

Hosoda K, Wang MF, Liao ML, Chuang CK, Iha M, Clevidence B, Yamamoto S (2003) Antihyperglycemic effect of oolong tea in type 2 diabetes. Diabetes Care 26(6):1714-1718

Jie G, Lin Z, Zhang L, Lv H, He P, Zhao B (2006) Free radical scavenging effect of $\mathrm{Pu}$-erh tea extracts and their protective effect on oxidative damage in human fibroblast cells. J Agric Food Chem 54(21):8058-8064

Karori S, Wachira F, Wanyoko J, Ngure R (2010) Antioxidant capacity of different types of tea products. Afr J Biotechnol 6(19):22872296
Kondo S, Tsuda K, Muto N, Ueda J (2002) Antioxidative activity of apple skin or flesh extracts associated with fruit development on selected apple cultivars. Sci Hortic-Amsterdam 96(1-4):177185

Kusano R, Andou H, Fujieda M, Tanaka T, Matsuo Y, Kouno I (2008) Polymer-like polyphenols of black tea and their lipase and amylase inhibitory activities. Chem Pharm Bull 56(3):266-272

Layer P, Rizza RA, Zinsmeister AR, Carlson GL, Dimagno EP (1986) Effect of a purified amylase inhibitor on carbohydrate tolerance in normal subjects and patients with diabetes mellitus. Mayo Clin Proc 61(6):442-447

Ling TJ, Wan XC, Ling WW, Zhang ZZ, Xia T, Li DX, Hou RY (2010) New triterpenoids and other constituents from a special microbial-fermented Tea-Fuzhuan brick tea. J Agric Food Chem 58(8):4945-4950

Loo AE, Huang D (2007) Assay-guided fractionation study of $\alpha$ amylase inhibitors from Garcinia mangostana pericarp. J Agric Food Chem 55(24):9805-9810

Ludwig DDS (2002) The glycemic index - physiological mechanisms relating to obesity, diabetes, and cardiovascular disease. Jama-J Am Med Assoc 287(18):2414-2423

Matsumoto N, Tonooka F, Ishigaki A. Hara Y (1991) Reduction of blood-glucose level by catechins. Proceedings of the International Symposium on Tea Science 318-321

Miliauskas G, Venskutonis P, Van Beek T (2004) Screening of radical scavenging activity of some medicinal and aromatic plant extracts. Food Chem 85(2):231-237

Moussa SA (2008) Oxidative stress in diabetes mellitus. Romanian J Biophys 18(3):225-236

Notkins AL (2002) Immunologic and genetic factors in type 1 diabetes. J Biol Chem 277(46):43545-43548

Ponnusamy S, Ravindran R, Zinjarde S, Bhargava S, Kumar AR (2011) Evaluation of traditional Indian antidiabetic medicinal plants for human pancreatic amylase inhibitory effect in vitro. Evid Based Complement Alternat 2011:1-10

Shi J, Kakuda Y (2006) Herbs: challenges in chemistry and biolog. Oxford University Press, Oxford

Siebert KJ, Troukhanova NV, Lynn PY (1996) Nature of polyphenolprotein interactions. J Agric Food Chem 44(1):80-85

The first research institute of China Standards Publisher (2003) Standards collection for tea (Chinese). China Standards Publisher, Beijing

Thompson LU, Yoon JH (1984) Starch digestibility as affected by polyphenols and phytic acid. J Food Sci 49(4):1228-1229

Yang XQ (2003) Tea polyphenol chemistry (Chinese). Shanghai Science and Technology Press, Shanghai

Yoon JH, Thompson LU, Jenkins D (1983) The effect of phytic acid on in vitro rate of starch digestibility and blood glucose response. Am J Clin Nutr 38(6):835-842

Zhang DY, Shao WF, Liu ZH, Huang YW, Shi ZP (2009) Research on the chemical constituents of Pu-erh tea and its inhibition effect on $\alpha$-amylase (Chinese). Southwest China J Agric Sci 22(1):52-54

Zhang L, Li N, Ma ZZ, Tu PF (2011) Comparison of the chemical constituents of aged $\mathrm{Pu}$-erh tea, ripened $\mathrm{Pu}$-erh tea, and other teas using HPLC-DAD-ESI-MS(n). J Agric Food Chem 59(16):87548760

Zhong L, Furne JK, Levitt MD (2006) An extract of black, green, and mulberry teas causes malabsorption of carbohydrate but not of triacylglycerol in healthy volunteers. Am J Clin Nutr 84(3):551-555

Zhou DR, Chen YQ, Ni DJ (2009) Effect of water quality on the nutritional components and antioxidant activity of green tea extracts. Food Chem 113(1):110-114

Zhu Y, Huang H, Tu Y (2006) A review of recent studies in China on the possible beneficial health effects of tea. Int J Food Sci Technol 41(4):333-340

Zunino SJ (2009) Type 2 diabetes and glycemic response to grapes or grape products. J Nutr 139(9):1794-1800 